\title{
Flexible Distributed Multi-Energy Generation System Expansion Planning under Uncertainty
}

\author{
E. A. Martinez Cesena, Member, IEEE, T. Capuder, Member, IEEE, \\ and P. Mancarella, Senior Member, IEEE
}

\begin{abstract}
A key feature of smart grids is the use of demand side resources to provide flexibility to the energy system and thus increase its efficiency. Multi-energy systems where different energy vectors such as gas, electricity and heat are optimised simultaneously prove to be a valuable source of demand side flexibility. However, planning of such systems may be extremely challenging, particularly in the presence of long-term price uncertainty in the underlying energy vectors. In this light, this work proposes a unified operation and planning optimisation methodology for Distributed Multi-energy Generation (DMG) systems with the aim of assessing flexibility embedded in both the operation and investment stages subject to long-term uncertainties. The proposed approach reflects Real Options thinking borrowed from finance, and is cast as a stochastic Mixed Integer Linear Programme. The methodology is illustrated through a realistic UK based DMG case study for district energy systems, with Combined Heat and Power plant, Electric Heat Pumps, and Thermal Energy Storage. The results show that the proposed approach allows reduction in both expected cost and risk relative to other less flexible planning methods, thus potentially enhancing the business case of flexible DMG systems.
\end{abstract}

Index Terms - Distributed generation, flexibility, multienergy systems, planning under uncertainty, real options, risk, storage.

\section{NOMENCLATURE}

\section{Decision variables}

$\begin{array}{lll}C & \text { Total cost of the system } & (£) \\ C_{o} & \text { Operational cost of the system } & (£ / \text { year }) \\ D_{\text {des }} & \text { Installed CHP, EHP and TES capacity } & (\mathrm{kW}) \\ E_{C H P} & \text { CHP electrical export } & \left(\mathrm{kW}_{\mathrm{e}}\right) \\ E_{E H P} & \text { EHP electrical export } & \left(\mathrm{kW}_{\mathrm{e}}\right) \\ E_{\text {exp }} & \text { Plant electrical export to the grid } & \left(\mathrm{kW}_{\mathrm{e}}\right) \\ E_{\text {imp }} & \text { Plant electrical import from the grid } & \left(\mathrm{kW}_{\mathrm{e}}\right) \\ F_{\text {aux }} & \text { Boiler fuel input } & \left(\mathrm{kW}_{\mathrm{th}}\right) \\ F_{C H P} & \text { CHP fuel input } & \left(\mathrm{kW}_{\mathrm{th}}\right) \\ H_{a u x} & \text { Boiler heat output } & \left(\mathrm{kW}_{\mathrm{th}}\right) \\ H_{C H P} & \text { CHP heat output } & (\mathrm{kW} \text { th } \\ H_{E H P} & \text { EHP heat output } & (\mathrm{kW} \\ H_{S} & \text { TES accumulated heat } & (\mathrm{kWh}) \\ I_{C H P} & \text { CHP binary status (on or off) } & \in[0,1] \\ I_{d e s} & \text { Investment decision } & \in[0,1] \\ \text { Input parameters } & \\ C_{I} & \text { Cost of investment } & (£) \\ C O P & \text { EHP coefficient of performance } & \\ C_{O \& M} & \text { CHP operation and maintenance cost } & \left(£ / \mathrm{kWh}_{\mathrm{e}}\right) \\ D e s & \text { Feasibility of a capacity upgrade } & \in[0,1] \\ D F_{I} & \text { Factor for discounting investments } & \\ D F_{o} & \text { Factor for discounting operational costs } & \\ D g_{\text {des }} & \text { Predefined design (multi-stage constraint) } & \in[0,1]\end{array}$

\begin{tabular}{|c|c|c|}
\hline$d t$ & Length of a time period & (h) \\
\hline$E_{\text {CHP_max }}$ & CHP electrical capacity & $\left(\mathrm{kW}_{\mathrm{e}}\right)$ \\
\hline$E_{\text {CHP_min }}$ & CHP minimum electrical output & $\left(\mathrm{kW}_{\mathrm{e}}\right)$ \\
\hline$E_{D}$ & Electrical demand & $\left(\mathrm{kW}_{\mathrm{e}}\right)$ \\
\hline$H_{\text {aux_max }}$ & Boiler capacity & $\left(\mathrm{kW}_{\mathrm{th}}\right)$ \\
\hline$H_{D}$ & Heat demand & $\left(\mathrm{kW}_{\mathrm{th}}\right)$ \\
\hline$H_{E H P \_\max }$ & EHP capacity & $\left(\mathrm{kW}_{\mathrm{th}}\right)$ \\
\hline$H_{S_{-} \max }$ & TES capacity & $\left(\mathrm{kWh}_{\mathrm{th}}\right)$ \\
\hline$H_{S \_ \text {min }}$ & TES minimum storage level & $\left(\mathrm{kWh}_{\mathrm{th}}\right)$ \\
\hline$P_{\text {Eimp }}$ & Price of electrical import from the grid & $\left(£ / \mathrm{kWh}_{\mathrm{e}}\right)$ \\
\hline$P_{\text {Eexp }}$ & Price of electrical export to the grid & $\left(£ / \mathrm{kWh}_{\mathrm{e}}\right)$ \\
\hline$P_{\text {gas }}$ & Price of gas & $\left(£ / \mathrm{kWh}_{\mathrm{th}}\right)$ \\
\hline Prob & Probability of occurrence & $(\% / 100)$ \\
\hline$r m p$ & CHP electrical ramp & $\left(\mathrm{kWh}_{\mathrm{e}}\right)$ \\
\hline$\eta_{1 e}, \eta_{2 e}$ & CHP electrical efficiency curve components & \\
\hline$\eta_{1 t}, \eta_{2 t}$ & CHP thermal efficiency curve components & \\
\hline$\eta_{\text {aux }}$ & Boiler efficiency & \\
\hline$\eta_{S}$ & TES efficiency & \\
\hline \multicolumn{3}{|l|}{ Indices } \\
\hline$a$ & Investment alternatives & \\
\hline$g$ & Time period before/after multi-stage constrai & \\
\hline $\operatorname{Nod}(s, y)$ & Tree node corresponding to $s$ and $y$ & \\
\hline$p$ & Design parameter & \\
\hline$s, s_{1}, s_{2}$ & Scenarios & \\
\hline$t$ & Time period (operation) & \\
\hline$y$ & Time period (investment) & \\
\hline
\end{tabular}

\section{INTRODUCTION}

TNDER the smart grid paradigm, the existence of information and communication technologies is expected to facilitate the coordinated operation, control and optimisation of multiple demand side resources [1]. This is particularly beneficial for the emergence of multi-energy systems that integrate and optimise the use of different energy vectors such as gas, electricity and heat, among others [2]. The deployment of multi-energy systems in smart grids is seen as a key option to provide flexibility to the energy system and improve its overall efficiency and environmental performance.

In this general context, the concept of Distributed Multienergy Generation (DMG) [3], in particular, has wide potential applications such as for district heating [4], commercial buildings/hospitals [5], and so on.

DMG systems comprise a combination of different technologies capable of coupling different energy vectors and offer higher efficiencies than conventional "separate" electricity and heat generation. In the case of Combined Heat and Power (CHP) [6][7], in case coupled to Electric Heat Pumps (EHP) [8] and Thermal Energy Storage (TES), these 
benefits are known from the operational and environmental point of view. And while single CHP and EHP units are characterized by high efficiency but low flexibility in responding to system requirements, complex DMG structures where these units are coupled can bring substantial economic benefits in terms of optimal trade-off between available energy vectors [9][10]. In addition, coupling CHP-TES or CHP-EHP has proven to have the potential to significantly reduce global $\mathrm{CO}_{2}$ emissions [11] and result in primary energy savings [8][12][13]. These benefits from coupling the operation of different DMG technologies can be called, operational flexibility.

DMG plants composed of different multi-energy technologies could thus bring significant system benefits. However, their planning may be exposed to substantial risk, particularly under realistic conditions characterised by significant uncertainties in energy prices. This motivates the implementation of advanced risk hedging tools such as flexible planning methodologies facilitated by Real Options (RO) thinking [14], which could boost the business case for DMG systems. In fact, the use of RO thinking, borrowed from finance, for the purpose of investment planning in engineering systems has the desirable attributes of minimising risks from negative scenarios and maximising benefits from positive ones by capturing the value of flexibility in investment decisions (e.g., defer or accelerate investments) and system design (e.g., undersize the plant at an initial stage and increase its capacity whenever needed) [15].

However, there is still little understanding and quantification of the aforementioned concepts, particularly of the implications from coupling of different technologies and energy vectors, which represent ongoing research from many perspectives. For instance, the majority of literature investigates the operation of DMG systems with predefined technologies in a deterministic scenario [11][16], optimal investments without flexibility [17][18], or RO based investments under uncertainty for single technology DMG systems [19][20]. Other studies have addressed the operation of DMG plants that couple various technologies in deterministic scenarios [21-23], investment under uncertainty for multi-technology DMG plants in a RO context [24], or single DMG technology investment according to different decision criteria in a comprehensive multi-temporal uncertainty framework [25][26]. However, none of these including investment time flexibility. Available literature on investment planning applied to other areas (e.g., transmission network planning and Phase Measurement Unit placement), highlight that multi-stage algorithms can be used to model investment timing flexibility (sometimes by combining the approach with RO thinking) as long as the associated search space is tractable [27-30]. For instance, in [27] a multi-stage algorithm is combined with the concept of options to assess flexibility in the form of options that may become available after a transmission expansion, while maintaining the problem tractable by only considering two decision stages and a simplified model of the network. Other studies recommend the use of scenario reduction techniques or heuristic approaches to limit the search space, which may not guarantee finding the optimal solutions (e.g., by disregarding impacts of immediate decisions on the value of future investments) [28-30].

In this light, there are clear research gaps relevant to DMG planning under uncertainty including flexibilities in operation, multi-technology selection, and investment timing.

In the light of this, this work presents a new methodology for unified flexible operational and planning optimisation of DMG systems subject to long-term uncertainty. The framework has been devised to specifically address different types of potential DMG flexibilities, namely (i) operational flexibility in optimally responding to energy market signals from an operational perspective and (ii) investment flexibility in responding to long-term evolution of energy prices through optimal selection of DMG equipment type and size as well as relevant investment timing. More specifically, the methodology can capture operational flexibility brought about by the interaction between multi-energy technologies (e.g., CHP, EHP, and TES) and arbitrage between different energy vectors expressed with a specifically developed generalised Mixed Integer Linear Programming (MILP) formulation. Moreover, the results from the operational optimisation module are embedded in a flexible planning engine whereby investment decisions are cast as a stochastic programming problem based on RO thinking and formulated as a MILP, which allows the methodology to capture investment flexibility value too.

The proposed approach naturally provides an investment risk hedging strategy and avoids investment lock-out, as it will be explored in detail throughout this work. Other planning philosophies including the traditional use of forecasts ("best view") or scenario trees ("multi-stage") are also incorporated and analysed as sub-cases of the proposed model with the objective of highlighting how different planning philosophies capture (or disregard) flexibility value and as a benchmark for the benefits of the approach introduced here.

The rest of the paper is structured as follows. Section II presents the DMG system configuration considered for this study, which represents a general DMG system for district energy system applications coupling Boiler, CHP, EHP, and TES technologies. A novel generalised approach to map the energy flows of different system configurations is also presented, which will allow a compact and unified formulation of the proposed optimal flexible operational and investment methodology, described in Section III. The key attributes of the approach are illustrated with a realistic UK based case study in Section IV. The main findings and conclusions of this work are then summarised in Section V.

\section{DMG SYSTEM GENERAL CONFIGURATION}

Typical applications of DMG plants are for district energy systems in which gas, electricity and heat vectors optimally interact to meet the energy demands of the district energy system's customers. In this work, it is assumed that this type of DMG plant can be composed of any combination of Boiler, CHP, EHP and TES units, which represent the majority of technologies typically available for such applications. As a 


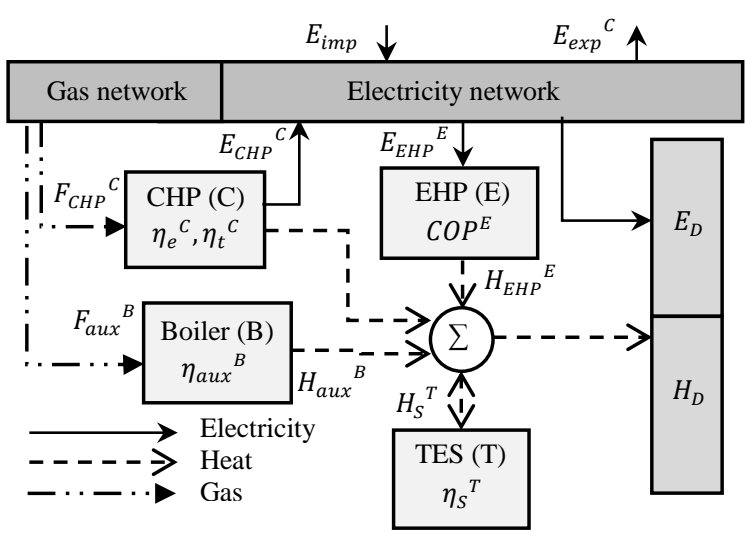

Fig. 1. Generalized flow diagram of the DMG system.

result, plentiful DMG designs given by type (and size) combinations of these technologies can be devised. Hence, as mentioned earlier, rather than mapping all the potential energy flows in the different configurations for a handful of cases, a generalised flow diagram capable of mapping all possible combinations has been developed and is presented in Fig. 1.

The generalised flow diagram relies on superscripts to denote the elements of the system that must be mapped when designing a given DMG configuration with Boiler (B), CHP (C), EHP (E) and/or TES (T) devices. In addition, as the diagram is generalised, other technologies can be included by choosing a proper superscript, formulating the particular elements and plugging them into the flow diagram. In order to model a specific DMG system configuration, the elements with relevant superscripts are included into the model. For example, the flow diagram of a BET system comprising Boiler, EHP and TES units would be modelled with all elements with the superscripts $\mathrm{B}, \mathrm{E}$ and $\mathrm{T}$ (i.e., by removing elements with the $\mathrm{C}$ superscript). Note the elements without superscripts should always be included in the formulation of the DMG system.

\section{Methodology}

This section describes the RO thinking based methodology proposed in this work. A description of the methodology can be seen at a high level in Fig. 2 and is detailed below. It is important to note that the term RO thinking is used to refer to the flexibility embedded in the operation and investment of engineering projects, which may not be properly captured with classical RO tools (e.g., partial differential equations and dynamic programming, among others). Such type of flexibility can be assessed with traditional planning tools such as mathematical programming as long as the sources of flexibility are identified (e.g., by screening for options) and uncertainty is properly modelled (e.g., with non-recombining scenario trees) [14][31].

\section{A. Pre-processing}

At the pre-processing stage, all relevant inputs for the study are collected, namely, technical and economic inputs, and scenarios considered for modelling uncertainty.

The technical inputs include the characteristics of the
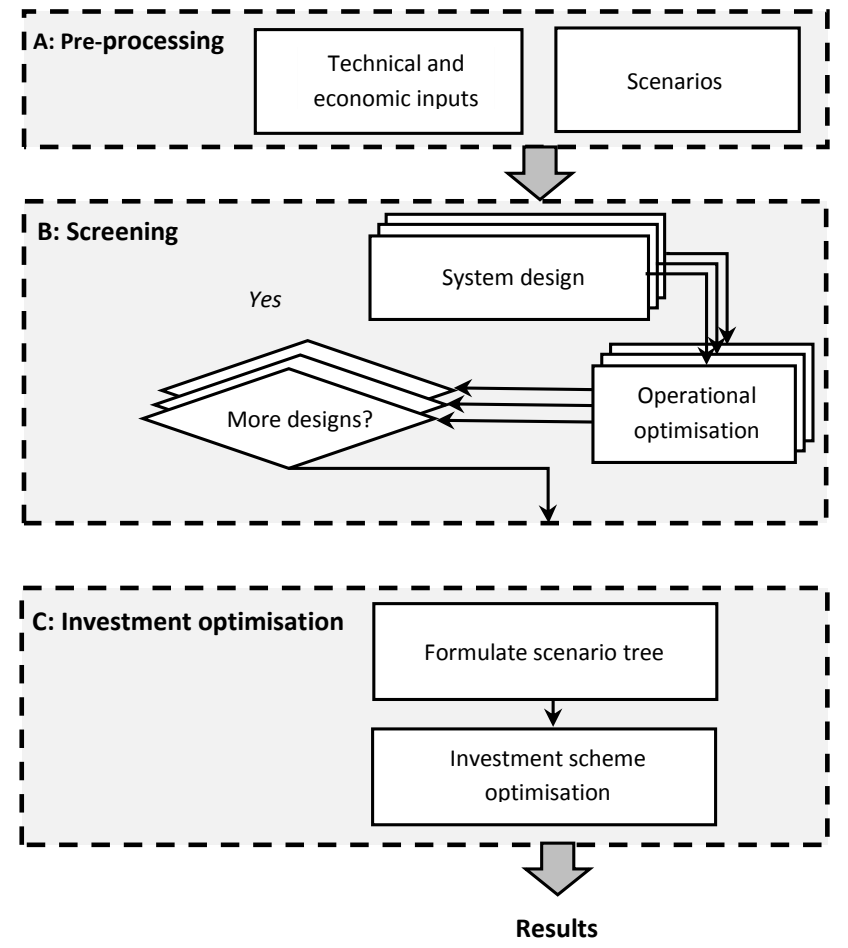

Fig. 2. Flow diagram of the proposed methodology.

considered equipment (e.g., capacity, cost and efficiency, among others). Providing these characteristics by technology and size allows the methodology to account for economies and efficiencies of scale (for instance, larger plants may feature higher efficiencies).

The economic inputs include the discount rate, planning horizon and other relevant parameters. These parameters are used to calculate the Net Present Cost (NPC) of the DMG systems under the assumption that all devices are replaced indefinitely at the end of their lifetime. This allows like-forlike comparison of DMG systems that may be upgraded (e.g., by installing additional units) in different scenarios/times and may thus have different lifetimes.

The different uncertainties (and in case their interactions/combinations) are modelled with a series of scenarios that are simulated at the following stage of the methodology. The amount of scenarios required to properly model uncertainty is a function of the characteristics of the uncertainty sources (e.g., model and correlations if any) and the planning horizon, and ultimately by the preferences of decision makers regarding trade-off between accuracy levels and computational time. In fact, both accuracy and computational time increase with the amount of scenarios considered. However, while assessing a large amount of scenarios is generally acceptable for planning studies even these were to take considerable computational time (e.g., several days), in most practical applications the amount of realistic future scenarios may be limited and what-if sensitivity studies may be preferred.

\section{B. Screening}

At this stage, a screening method based on RO thinking and 
exhaustive searches has been selected, while a unified MILP optimisation routine is used to calculate the related operational costs in the considered scenarios and DMG configurations.

The literature provides several screening techniques based on RO thinking which can be used for the screening of flexibility in projects subject to computational, social, political and other constraints [32]. Among available screening approaches, the exhaustive search was selected as the search space associated with the DMG designs considered in this work is sensible, as will be further discussed in the next section (case study). The operation of each DMG system under consideration is explored by modelling the system as the generalised MILP problem denoted by (1) - (12):

$$
\begin{aligned}
& \text { minimise: } \\
& \begin{aligned}
C_{o} & =d t \times \sum_{t}\left[P_{\text {gas }}(t) \times\left(F_{\text {aux }}(t)^{B}+F_{C H P}(t)^{C}\right)\right. \\
& +C_{O \& M}(t)^{C} \times E_{C H P}(t)^{C} \\
& \left.+P_{\text {Eimp }}(t) \times E_{\text {imp }}(t)-P_{\text {Eexp }}(t)^{C} \times E_{\text {exp }}(t)^{C}\right]
\end{aligned}
\end{aligned}
$$

s.t.

$$
\begin{aligned}
& 0 \leq H_{\text {aux }}(t)^{B} \leq H_{\text {aux_max }}{ }^{B} \\
& H_{\text {aux }}(t)^{B}=\eta_{\text {aux }}(t)^{B} \times F_{\text {aux }}(t)^{B} \\
& I_{\text {CHP }}(t)^{C} \times E_{\text {CHP_min }}{ }^{C} \leq E_{\text {CHP }}(t)^{C} \\
& \leq I_{C H P}(t)^{C} \times E_{\text {CHP_max }}{ }^{C} \\
& E_{C H P}(t)^{C}=F_{C H P}(t)^{C} \times \eta_{1 e^{C}}+\eta_{2 e^{C}} \times I_{C H P}(t)^{C} \\
& -r m p^{C} \times d t \leq E_{C H P}(t)^{C}-E_{C H P}(t-1)^{C} \\
& \leq r m p^{C} \times d t \\
& H_{C H P}(t)^{C}=F_{C H P}(t)^{C} \times \eta_{1 t}(t)^{C}+\eta_{2 t}{ }^{C} \\
& \times I_{C H P}(t)^{C} \\
& 0 \leq H_{E H P}(t)^{E} \leq H_{E H P \_ \text {max }}{ }^{E} \\
& H_{E H P}(t)^{E}=E_{E H P}(t)^{E} \times C O P(t)^{E} \\
& H_{S_{-} \text {min }}{ }^{T} \leq H_{S}(t)^{T} \leq H_{S_{-} \text {max }}{ }^{T} \\
& H_{D}(t) \leq\left(\eta_{S} \times H_{S}(t-1)^{T}-H_{S}(t)^{T}\right) / d t \\
& +H_{\text {aux }}(t)^{B}+H_{C H P}(t)^{C}+H_{E H P}{ }^{E} \\
& E_{D}(t)=E_{\text {imp }}(t)+E_{C H P}(t)^{C}-E_{\text {exp }}(t)^{C} \\
& -E_{E H P}(t)^{E}
\end{aligned}
$$

Equations (1) - (12) offer a generalised mathematical model for the operation of DMG systems based on the same rationale proposed for the generalised flow diagram presented in Fig. 1. That is, a particular system design (e.g., BCT) is denoted by the elements with the corresponding superscripts (e.g., B, C and T), as well as all elements without a superscript. More specifically, the objective function in (1) is to minimise operational costs (NPC in this work) from fuel and electricity imports while benefiting from energy exports, by exploiting arbitrage between electricity and gas prices. The capacity limit and operation of Boilers are denoted by (2) and (3), respectively. Equations (4) - (7) model CHP. Maximum CHP electricity generation capacity is denoted by (4), whereas electricity output subject to an efficiency curve and ramp up/down constraints are modelled with (5) and (6), respectively. The CHP heat output subject to an efficiency curve is denoted by (7). Equations (8) and (9) denote the capacity limits and operational efficiency of EHP units, respectively. The storage limits of TES are modelled with (10). Heat and Electricity balance is imposed by (11) and (12), respectively. It is worth emphasizing that DMG systems have the flexibility to meet the balance equations while minimising costs by coupling different technologies that, for instance, provide alternatives to supply demand or other devices (e.g., CHP), produce heat from fuel or electricity (e.g., CHP and EHP), produce electricity from fuel, and store surplus heat for later use (e.g., TES). This operational flexibility becomes valuable if the spark spread between gas and electricity or the time-shift characteristics of TES can be exploited.

Operational optimisation simulations are run on a half hourly basis for a yearly interval, and it is assumed that the simulated conditions are representative of the whole period between two consecutive scenarios.

\section{Investment optimisation}

At this stage, a flexible investment scheme is determined considering the operational costs calculated in the previous stage, and RO as well as other planning philosophies.

In order to capture the value of flexible investment decisions in DMG, while also incorporating the value of flexible operation analysed in the previous stage, a stochastic programming model constrained by a customised nonrecombining scenario tree is proposed. Such an approach can identify the value embedded in the design of engineering systems [15]. This is owing to the path dependencies introduced by the non-recombining tree, which allows the methodology to hedge against the risk of investment lock-out. For example, after investing in a $500 \mathrm{~kW}_{\mathrm{e}}$ CHP unit, path dependency forces DMG designs in future dependent scenarios to include the $500 \mathrm{~kW}_{\mathrm{e}} \mathrm{CHP}$ unit. Based on this, the stochastic programming model denoted by (13) - (18) is proposed to identify flexible investments in DMG systems.

$$
\begin{aligned}
C & =\sum_{s, y}^{\operatorname{minimise}} \operatorname{Prob}(s) \times\left\{D F_{o}(y) \times \sum_{a} C_{o}(s, y, a)\right. \\
& \times I_{\text {des }}(s, y, a)+D F_{I}(y) \times \sum_{p} C_{I}(p) \\
& \left.\times\left[D_{\text {des }}(s, y, p)-D_{\text {des }}(s, y-1, p)\right]\right\}
\end{aligned}
$$

s.t.

$$
\begin{gathered}
D_{\text {des }}(s, 0, p)=0 \\
1=\sum_{a} I_{\text {des }}(s, y, a) \\
D_{\text {des }}(s, y, p)=\sum_{a} I_{\text {des }}(s, y, a) \times \operatorname{Des}(a, p) \\
D_{\text {des }}(s, y+1, p) \geq D_{\text {des }}(s, y, p)
\end{gathered}
$$




$$
\begin{aligned}
D_{\text {des }}\left(s_{1}, y, p\right) & =D_{\text {des }}\left(s_{2}, y, p\right), \\
& \forall s_{1}, s_{2} \mid \operatorname{Nod}\left(s_{1}, y\right)=\operatorname{Nod}\left(s_{2}, y\right)
\end{aligned}
$$

The objective function in (13) aims at minimising expected costs from investments in DMG technologies and operation throughout the scenario tree. In this work, costs are quantified based on the NPC criteria; hence, investment costs are discounted with a factor that reflects investment timing (including additional investments to replace the units at the end of their lifetime) and operational costs are discounted with a factor that reflects both timing and periodicity (it is assumed that operational costs are the same within periods between scenarios, as mentioned above). Based on this objective function, the model seeks series of flexible investments in response to the evolution of uncertainty, avoiding risks from investment lock-out that may result in significantly oversized or undersized systems after the unfolding of uncertainty. The feasibility of the different investment decisions is enforced with (14) - (17). That is, a new DMG system may be built (14), based on a series of decisions (15) regarding the most cost effective and feasible system upgrades available (16) (e.g., including doing nothing or install a given device) subject to the characteristics of the current system (17). It is important to note that the design parameters $(p)$ is used to denote different devices, which can belong to the same technology. For example, the design parameters can be used to represent $500 \mathrm{~kW}_{\mathrm{e}} \mathrm{CHP}, 1 \mathrm{MW}_{\mathrm{e}} \mathrm{CHP}, 2 \mathrm{MW}_{\mathrm{th}}$ EHP, and so on. This allows the algorithm to discriminate DMG systems with the same capacity but different devices (e.g., one $1 \mathrm{MW}_{\mathrm{e}} \mathrm{CHP}$ and two $500 \mathrm{~kW}_{\mathrm{e}}$ units) and formulate feasible design upgrades that include devices already installed in the system. Finally, (18) imposes the nonanticipativity constraints required to formulate the non-recombining scenario tree. That is, the decision variables in scenarios that share the same previous information (i.e., scenarios that are identical until a given period) are forced to be the same; hence, realistic investment decisions are enforced based on past information and forecasts of plausible futures instead of on perfect information.

Finally, (19) and (20) are included into the methodology to model typical planning philosophies other than RO:

$$
\begin{array}{rr}
D_{\text {des }}(s, y, p)=D g_{\text {des }}(s, y, p), & \forall y \mid y<g \\
D_{\text {des }}(s, y, a)=D_{\text {des }}(s, y-1, a), & \forall y \mid y>g
\end{array}
$$

In fact, in this work three planning philosophies besides RO are considered, namely (i) not investing in a DMG system (donothing), (ii) investing immediately in a DMG system based on a forecasts (best view) and (iii) investing immediately in a DMG system based on the full tree and upgrading the system based on a rolling horizon (multi-stage). In practice, the best view philosophy may be the most commonly used, whereas the multi-stage philosophy may be one of the best techniques available to capture the value of flexibility. In this case, the multi-stage approach is combined with a heuristic tool to allow the algorithm to consider all scenarios with the cost of disregarding potential impacts of immediate investments on future decisions for the relevant simplification).
The do-nothing philosophy is modelled by using (19) to set the capacity of the DMG system equal to zero throughout the tree $\left(D g_{\text {des }}(s, y, p)=0\right)$. This philosophy does not involve any investments and does not capture any flexibility value.

The best view philosophy is modelled by replacing the tree with a single scenario (taken as the mean values in all scenarios corresponding to a particular time period) and setting $g=1$ in (20), which forces the optimiser to select a single DMG design that has to be implemented immediately (without considering that the DMG may be upgraded at a later stage). Once the best view design has been determined, it is assessed using the full scenario tree. This philosophy assumes that a single forecast is used to assess whether to invest in a DMG system design immediately or never. As a result, the philosophy may capture some operational flexibility value embedded in the DMG system. However, this value may be limited as the price differentials between energy vectors may not be properly modelled.

The multi-stage philosophy is modelled by solving the full stochastic programming model for every time period $(y)$, while locking previous investment decisions with (19) and neglecting future investment decisions with (20). As a result, this philosophy assumes that project planners will always optimise immediate investment decisions without considering future contingent investments. However, the project is revised and (if needed) upgraded in the future. Hence, this philosophy can capture the operational flexibility value embedded in the DMG systems, as well as some investment flexibility associated with the capability of project planners to upgrade the DMG system in response to the evolution of uncertainty. Investment flexibility is undervalued based on this philosophy, as current actions (taken without regard for future potential system upgrades) may prevent attractive system upgrades in the future, which is referred to as investment lock-out.

Finally, the proposed RO methodology is modelled with (13) - (18) (without including (19) and (20)). This philosophy assumes fully flexible investment decisions throughout the tree, which take into account previous decisions, as well as future potential system upgrades in response to the unfolding of uncertainty. Accordingly, the RO philosophy is meant to fully capture the value of operational and investment flexibility, which can be used to maximises benefits or minimise costs from scenarios that materialise via avoiding investment lock-out to facilitate low cost system upgrades. In this light, the proposed methodology can be used for the planning and design of DMG systems while capturing the value of flexibility, as well as for the assessment of flexibility value associated with different planning philosophies. Both of these applications will be explored below with a case study.

It is important to highlight that, ultimately, the proposed approach would make a recommendation for a first investment decision (including the option to wait), as well as a wide range of investment decisions contingent to the unfolding of uncertainty. The project planner may follow the recommendations for the first decision, as well as the contingent investments in the relevant scenario of the tree if 
such scenario is to materialize. However, in practice, the planner may be in a position to improve the models for uncertainty with time, in which case the methodology could be used periodically to produce improved recommendations for first (and contingent) investments.

\section{CASE STUDY}

\section{A. Case study description}

It is assumed that an investment in a DMG system for district energy system applications is being considered for a UK neighbourhood with roughly 1000 consumers with different heat and electricity consumption profiles. Currently heat demand is met by several Boilers with a total capacity of $6 \mathrm{MW}_{\text {th }}$. Typical electricity demand (ED) and heat demand (HD) consumption profiles for the neighbourhood during representative days are shown in Fig. 3, while base electricity profiles were taken from current UK market prices. These profiles represent the current condition of the neighbourhood, whereas future $\mathrm{ED}, \mathrm{HD}$, gas prices (GP) and electricity prices (EP) are deemed uncertain.

It is assumed that the DMG system can be designed with any combinations and number of the CHP, EHP and TES units shown in Table I, and that relevant smart grid infrastructure to couple, operate and optimise the units is available. That is, several units (or none) of each technology shown in Table I can be used in clusters to set up a DMG plant of desired capacity and type. Investments are assessed based on the NPC criterion at a $5 \%$ discount rate.

As discussed, uncertainty is modelled with a customisable non-recombining scenario tree. In this illustrative example, it is assumed that investment decisions can be made every 5 years during a planning horizon of 15 years and, after each decision node, electricity and gas consumption may remain the same (30\% probability) or increase by 5\% (70\% probability), whereas average electricity and gas prices may increase by $50 \%$ (60\% probability) or decrease by 50\% (40\% probability). That is, each decision node may span up to 16 scenarios. However, several branches of the tree are removed (introducing asymmetry), as prices above $200 \%$ and below $50 \%$ are deemed unlikely to occur. The resulting nonrecombining and asymmetric tree comprises 1600 scenarios and 1761 decision nodes. It is important to note that these parameters have been selected for illustrative purposes, as the tree can be customised as needed. Furthermore, the parameters used for the screening stage were defined based on exploratory analysis, which consisted on testing large DMG designs in extreme scenarios (i.e., combinations of smallest and largest values for each source of uncertainty) to estimate reasonable sizes to be considered for each technology. In practice, forecasts for relevant sources of uncertainty, socioeconomic conditions of the neighbourhoods, availability of energy resources in the area, available models for each technology and other studies, would be needed to define the parameters for the screening process.

Under the above-mentioned conditions, the proposed approach takes roughly 10 minutes to identify optimal investment decisions and DMG designs throughout the tree,
TABLE I. BASE CASE INDIVIDUAL DEVICES CONSIDERED FOR EACH TECHNOLOGY IN THE CASE STUDY

\begin{tabular}{|l|c|c|c|}
\hline Technology & Capacity & Efficiency & $\begin{array}{c}\text { Cost } \\
\left(£ \times 1 \mathbf{1}^{\mathbf{3}}\right)\end{array}$ \\
\hline CHP & $1000 \mathrm{~kW}_{\mathrm{e}}$ & $\begin{array}{c}35 \% \text { (electrical) } \\
45 \%(\text { thermal) }\end{array}$ & 500 \\
\hline EHP & $1000 \mathrm{~kW}_{\text {th }}$ & $3($ COP $)$ & 240 \\
\hline TES & $200 \mathrm{~m}^{3}$ & - & 140 \\
\hline
\end{tabular}

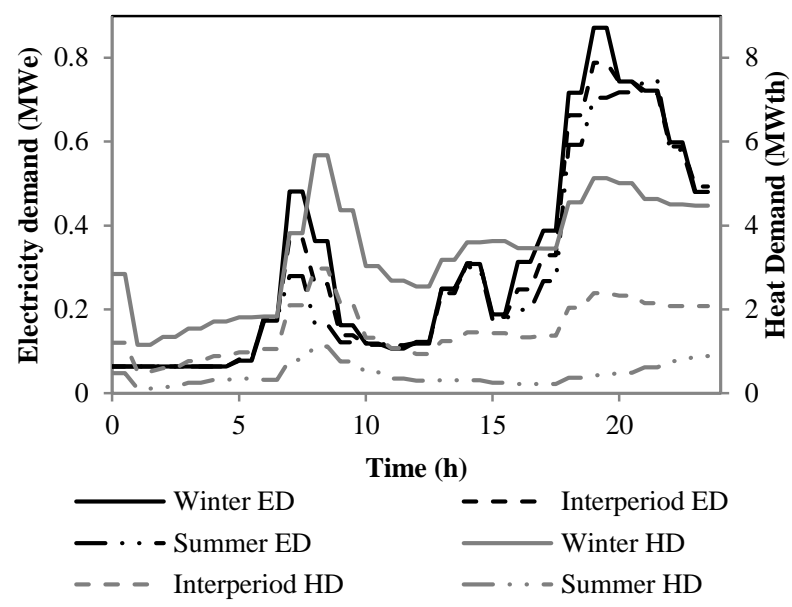

Fig. 3. Electricity and heat demand (ED and HD) for representative days.

while the screening process based on exhaustive searches takes roughly 6 hours to assess 225 design combinations in 81 scenarios when parallelised using 4 instances (using a computer with a i5-3470 $3.2 \mathrm{GHz}$ processor and 8GB RAM), which is clearly acceptable for planning purposes. Note that the operational costs of the different DMG designs in all scenarios throughout the non-recombining tree can be interpolated with an acceptable level of accuracy ( $2 \%$ error or lower) from the 81 scenarios considered at the screening stage. It is important to highlight that demand can be met with imports from the grid and the boiler in every scenario considered. As a result, there are no technical constraints that may impose investment decisions in given scenarios, which allows the study to focus on investments that may lead to lower energy costs.

While the entire tree has been analysed (see discussion and results below, from Table II on), for the sake of simplicity, the key features of the proposed methodology are illustrated with a small section of the whole tree, namely reduced tree (Fig. 4). The reduced tree comprises four scenarios corresponding to potential evolution of prices (i.e., EP and GP) and demand (i.e., ED, HD) from 5 to 15 years in the future. The scenarios were selected to illustrate the effects of demand growth (scenario 1460), and different combinations of differentials between electricity and gas prices (i.e., scenarios 1652, 1682 and 1686). Further discussion is provided below.

\section{B. Results and discussion}

The designs suggested by the RO and multi-stage planning philosophies (i.e., total installed CHP, EHP and TES capacity) throughout the reduced scenario tree are shown in Table II. The expected NPC of the DMG systems in every scenario of the reduced tree and throughout the complete scenario tree (i.e., NPC summation of the investments in the 1600 scenarios times the probability of occurrence of the respective scenario) 


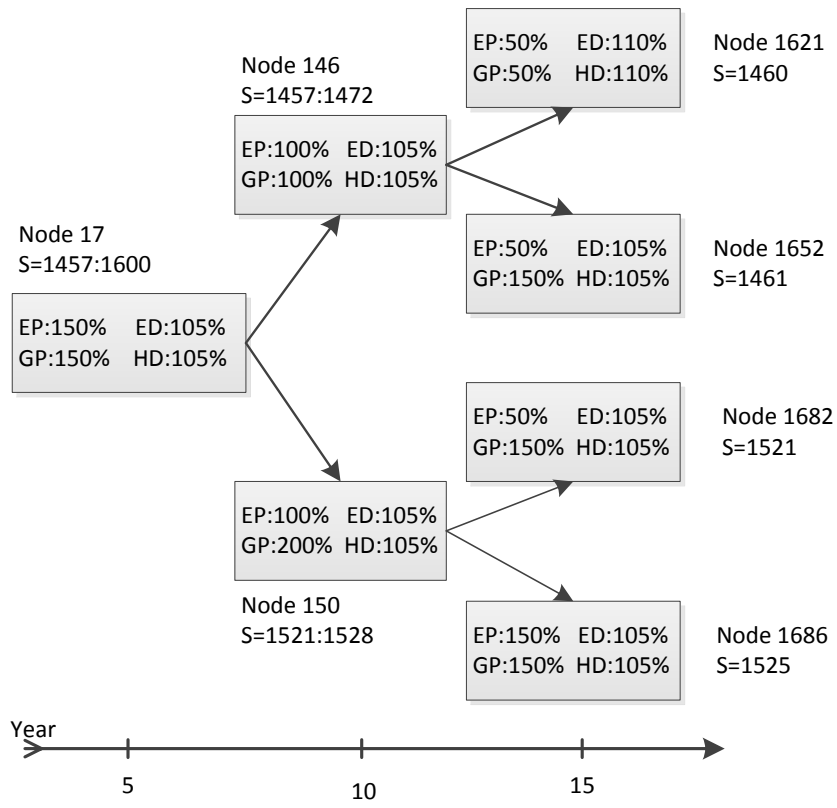

Fig. 4. Reduced scenario tree formed with a section of the nonrecombining scenario tree considered for this study.

based on all investment philosophies are shown in Table III.

The different scenarios in the reduced tree can drive investments in DMG systems of different sizes. For instance, it may be economically attractive to invest in large DMG systems whenever demand and prices increase significantly (e.g., scenario 1525), and when benefits can be gained from arbitrage (i.e., in scenarios 1652 and 1682), whereas small DMG systems may become more attractive under other conditions (e.g., scenario 1460). Accordingly, investment decisions in year 5 and 10 (i.e., nodes 17, 146 and 150) are challenging, as they may lead to sub-optimal designs in particular scenarios.

In this regard (and as can be seen in Table II) the recommendations of the $\mathrm{RO}$ and multi-stage philosophies are fundamentally different. On the one hand, the RO philosophy recommends investments in small DMG systems in year 5, which offer the flexibility to upgrade the system or hedge risks of oversizing it in specific scenarios, however may result in modest economic gain in the short-term. On the other hand, the multi-stage philosophy recommends medium-size systems in year 5, which may offer higher short-term benefits and may offer some flexibility to upgrade the system to maximise benefits in the right scenarios, but can lead to investment lockout.

An example of the conditions that may lead to investment lock-out for the multi-stage philosophy can be seen in scenario 1460. A small DMG design is attractive in this scenario as energy prices decrease constantly and evenly regardless of the demand increase in year 15. However, a medium size DMG system and a capacity upgrade are recommended respectively in nodes 17 and 146 (see Table II) to minimise energy costs in other scenarios, which results in high costs in scenario 1460 (i.e., from Table III, the multi-stage solution is more expensive that the best view solution in this scenario). The medium size DMG system recommended by the multi-stage philosophy in
TABLE II. DMG DESIGNS RECOMMENDED BY THE RO AND MULTI-STAGE

\begin{tabular}{|c|c|c|c|c|c|c|c|}
\hline \multirow{3}{*}{$\begin{array}{c}\text { Scenario } \\
\text { (s) }\end{array}$} & \multirow[b]{3}{*}{ Year } & \multicolumn{3}{|c|}{ APPROACHES } & \multirow{2}{*}{\multicolumn{3}{|c|}{ Multi-Stage }} \\
\hline & & & RO & & & & \\
\hline & & $\begin{array}{c}\text { CHP } \\
\left(\mathbf{k W}_{\mathrm{e}}\right)\end{array}$ & $\begin{array}{c}\text { EHP } \\
\left(\mathbf{k} W_{\text {th }}\right)\end{array}$ & $\begin{array}{l}\text { TES } \\
\left(\mathbf{m}^{3}\right)\end{array}$ & $\begin{array}{c}\text { CHP } \\
\left(k W_{e}\right)\end{array}$ & $\begin{array}{c}\text { EHP } \\
\left(\mathbf{k} \mathbf{W}_{\text {th }}\right)\end{array}$ & $\begin{array}{l}\text { TES } \\
\left(\mathbf{m}^{\mathbf{3}}\right)\end{array}$ \\
\hline \multirow{3}{*}{1460} & 5 & 1000 & 2000 & - & 3000 & 2000 & 200 \\
\hline & 10 & 1000 & 2000 & - & 3000 & 3000 & 400 \\
\hline & 15 & 1000 & 2000 & - & 3000 & $\overline{3000}$ & $\overline{400}$ \\
\hline \multirow{3}{*}{1461} & 5 & 1000 & 2000 & - & 3000 & 2000 & 200 \\
\hline & 10 & 1000 & 2000 & - & 3000 & 3000 & 400 \\
\hline & 15 & 1000 & $\underline{4000}$ & $\underline{\mathbf{2 0 0}}$ & 3000 & $\overline{4000}$ & $\overline{400}$ \\
\hline \multirow{3}{*}{1521} & 5 & 1000 & $\overline{2000}$ & - & 3000 & $\overline{2000}$ & 200 \\
\hline & 10 & 1000 & 4000 & 200 & 3000 & $\underline{3000}$ & 400 \\
\hline & 15 & 1000 & $\overline{4000}$ & $\overline{200}$ & 3000 & $\overline{\overline{4000}}$ & $\overline{400}$ \\
\hline \multirow{3}{*}{1525} & 5 & 1000 & 2000 & - & 3000 & $\overline{2000}$ & 200 \\
\hline & 10 & 1000 & 4000 & 200 & 3000 & 3000 & 400 \\
\hline & 15 & 1000 & 4000 & $\overline{400}$ & 3000 & 3000 & $\overline{400}$ \\
\hline
\end{tabular}

\begin{tabular}{|c|c|c|c|c|c|}
\hline \multirow{2}{*}{$\begin{array}{c}\text { Scenario } \\
(s)\end{array}$} & \multicolumn{4}{|c|}{ NPC $\left(£ \times 10^{3}\right)$} & \multirow{2}{*}{$\begin{array}{c}\text { Multi- } \\
\text { stage } \\
- \text { RO }(\%)\end{array}$} \\
\hline & $\begin{array}{c}\text { Do- } \\
\text { Nothing }\end{array}$ & $\begin{array}{l}\text { Best } \\
\text { view }\end{array}$ & $\begin{array}{c}\text { Multi - } \\
\text { stage }\end{array}$ & RO & \\
\hline 1460 & 10923 & 9715 & 9913 & 8680 & 12.4 \\
\hline 1461 & 15428 & 11123 & 10524 & 9340 & 11.2 \\
\hline 1521 & 16701 & 11698 & 10893 & 9570 & 12.1 \\
\hline 1525 & 18313 & 13358 & 13168 & 12459 & 5.4 \\
\hline $\begin{array}{l}1-1600 \\
\text { (Average) }\end{array}$ & 13049 & 9051 & 8673 & 7876 & 9.2 \\
\hline
\end{tabular}

years 5 and 10 is an attractive option in scenario 1525 as energy prices increase. In addition, further capacity upgrades are recommended in scenarios 1461 and 1521 to benefit from arbitrage that arises due to the different growth of EP (i.e., $50 \%$ ) and GP (i.e., 150\%).

As discussed above, the RO philosophy acknowledges the value of investment flexibility to customise the design of the DMG system for specific scenarios. As a result, it can be seen from Table II that a relatively smaller DMG system is recommended in year 5 to allow expansion customisation in every scenario that may materialise. This allows the RO philosophy to recommend the most economically attractive DMG designs in every scenario of the reduced tree and the complete tree, as shown in Table III. The results in Table III also show that the multi-stage philosophy is the second best option considering the full tree, followed by the best view philosophy. Considering that do-nothing philosophy is more expensive than other alternatives, it can be concluded that, in this case study, installing a DMG system is economically attractive.

Another key point to highlight is that investment flexibility does not simply increase the economic attractiveness of the DMG system by decreasing expected costs, but also by reducing risks. Actually, as shown in Fig. 5, the investment and operational flexibility value associated with some of the planning philosophies effectively shifts and skews (negative skewness) the corresponding expected NPC Probability Density Function (PDF) to the left. Thus flexibility value does not only reduce costs, but also limits the risks of incurring high costs in pessimistic scenarios. This is a well-known effect in $\mathrm{RO}$ literature, which considers that the risk of a project may be deemed low if the probability of incurring losses in 


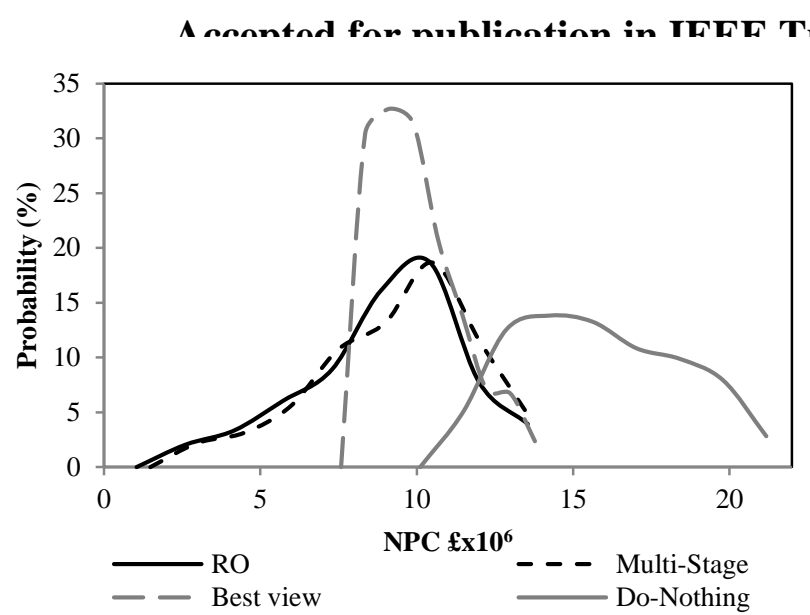

Fig. 5. NPC PDFs of investments planned under different philosophies throughout all scenarios.

pessimistic scenarios is reduced due to the effects of flexibility (regardless of the associated NPC standard deviation) [14][15][31].

The PDFs shown in Fig. 5 are consistent with discussion in the previous section regarding the flexibility value captured by each philosophy. The do-nothing alternative cannot capture any flexibility value, thus the corresponding NPC PDF is relatively normally distributed, as the distribution is defined by the scenarios considered in the tree (the fit improves for symmetric trees that comprise a large amount of scenarios). The best view philosophy only captures some marginal operational flexibility as future potential price differentials between energy vectors are underestimated. Thus, the corresponding NPC PDF only shows a marginal negative skewness. A comprehensive analysis on operational flexibility for DMG systems can be found in [13]. The study provides a detailed economic and environmental analysis of operational flexibility from coupling different types of DMG technologies (i.e., EHP, CHP and TES). It is shown that the economic benefits of operational flexibility from coupling DMG technologies are highly dependent on the cost of input fuel (in case gas) and market price of electricity. More precisely, in case of higher gas prices and of lower electricity prices, the option of CHP coupled with TES underperforms traditional boiler and grid option. This demonstrates insufficient flexibility CHP and TES option under specific market condition. Conducting the same analyses for coupled EHP, CHP, and TES these issues are alleviated resulting in savings of more than 50\% when compared with traditional boiler/electric grid provision of energy services. As those options have the possibility to dynamically optimize choice and quantity of their input fuel (in this context, dynamically is referred to time frame of market price changes every 30 minutes), they manage to avoid the pitfall of operating in periods when their marginal price is below that on the electricity market. Capability of using multiple input energy vectors results in high operational flexibility for all analysed cases in [13] manifested as operational cost savings ranging between 50 and $80 \%$ compared to procuring heat from gas boiler and electricity from the grid.

The multi-stage philosophy results in a NPC PDF with a significant negative skewness as it provides a better modelling

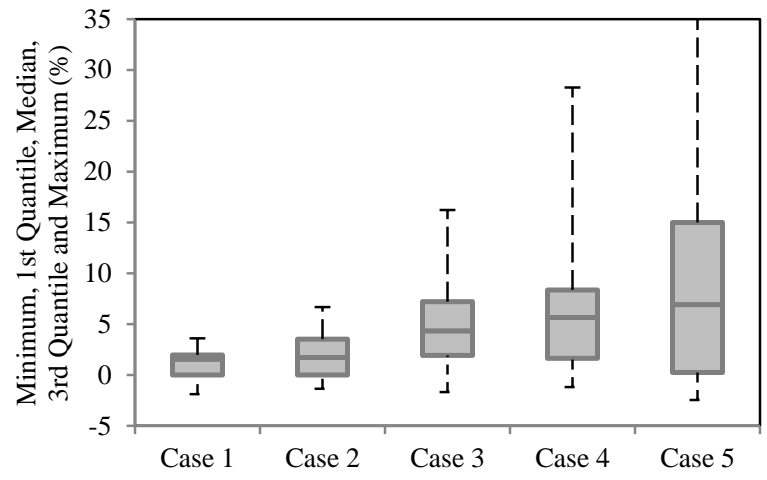

Fig. 6. Estimated flexibility value associated with changing from the bestview to the RO thinking philosophy.

of future expected price differentials and captures significant operational flexibility value and some investment flexibility value. The RO philosophy fully captures both operational and investment flexibility values and results in a NPC PDF that is significantly skewed and effectively shifted to the left.

Again with reference to Fig. 5, it is important to point out the capability of the methodology to analyse a large number of scenarios so as to generate PDFs. This is an attractive feature as the PDFs can be used to facilitate enhanced understanding and quantification of the different costs, flexibility and risk implications associated with different planning philosophies.

As a final remark, it is important to note that the value of flexibility as captured by the proposed methodology and underlying RO framework is a function of uncertainty. In fact, a RO based investment framework would not be able to capture significant value from flexibility in projects that are not subject to sufficient uncertainty. In order to illustrate this point, the case study was re-assessed for lower levels of uncertainty (i.e., assuming lower \pm variations to underlying uncertainty sources). The results, shown as box plots in Fig. 6, depict the median, and 25 and 75 percentiles of a NPC PDF corresponding to cost reductions associated with shifting from the best view to the RO planning philosophy (i.e., flexibility value captured by the RO philosophy that would be disregarded by the commonly used best view philosophy). Five cases are presented, in case one, the magnitude by which each uncertainty source varies per period was scaled by a factor of $1 / 5$ (e.g., prices and demand can vary by $\pm 10 \%$ and $\pm 1 \%$, respectively every time period), by a factor of $2 / 5$ in case 2 and so on.

It can be appreciated from Fig. 6 how reducing uncertainty levels may also reduce the economic value of flexible investment decisions, which indeed arises from its potential to hedge risks and minimise expected costs under uncertain scenarios. It can be seen that the proposed methodology is still attractive even under low uncertainty levels as RO thinking can still capture some operational and investment flexibility value, which may still bring significant economic benefits. Furthermore, the value of the proposed approach is clear in the face of substantial uncertainties that are expected in the future in energy markets as well as more generally (for instance, policy development, new technologies, and so forth). 


\section{CONCLUSION}

This work has proposed a unified flexible operation and planning optimisation methodology for DMG systems subject to long-term uncertainty. The novelties of the methodology include (i) an original generalised flow diagrams to map the energy flows of a wide range of different DMG systems and which allows synthetic writing of relevant equations; (ii) a new generalised MILP formulation for the optimisation of DMG systems that capture operational flexibility to respond to energy price signals by exploiting multi-vector arbitrage opportunities in the presence of different DMG technologies and time arbitrage opportunities in the presence of thermal storage (in case); and (iii) a stochastic programming model, again formulated as MILP, that allows type, size and investment time optimisation of multi-energy multicomponent flexible planning schemes considering long-term uncertainty and intrinsic risk hedging. Other investment philosophies are also incorporated in the proposed framework as sub-cases of the general flexible investment model.

This new methodology has been illustrated via a realistic UK DMG system case study for district energy system applications under a smart grid paradigm whereby underlying distributed resources can be coupled, controlled and optimised. The numerical results show that the flexible investment schemes produced with the RO thinking philosophy outperform investments recommended by other planning practices in terms of both expected costs and risks. It has been discussed how the use of RO based frameworks for the unified optimisation of the planning and operation of DMG systems can increase their economic attractiveness, particularly when subject to high levels of uncertainty.

\section{REFERENCES}

[1] P. Nyeng and J. Ostergaard, "Information and communications systems for control-by-price of distributed energy resources and flexible demand," IEEE Trans. Smart Grid, vol. 2, no. 2, pp.334 -341, 2011.

[2] P. Mancarella, "MES (multi-energy systems): an overview of concepts and evaluation models", Energy, vol. 65, pp. 1-17, 2014.

[3] G. Chicco, P. Mancarella, "Distributed multi-generation: A comprehensive view", Renew. Sustain. Energy Rev. vol. 13, pp. 535 $551,2009$.

[4] T. Nuytten, B. Claessens, K. Paredis, J. Van Bael, and D. Six, "Flexibility of a combined heat and power system with thermal energy storage for district heating," Appl. Energy, vol. 104, pp. 583 -591, 2013.

[5] G. Chicco and P. Mancarella, "From cogeneration to trigeneration: profitable alternatives in a competitive market, IEEE Trans. Energy Convers, vol.21, no.1, pp.265-272, 2006.

[6] J. H. Horlock, Advanced Gas Turbine Cycles. Elsevier, 2003, pp. 1 230.

[7] X. Zhang, G. G. Karady, K. R. Piratla, S. T. Ariaratnam, "Network capacity assessment of combined heat and power-based distributed generation in urban energy infrastructures," IEEE Trans. Smart Grid, vol. 4, no. 4, pp. $2131-2138,2013$.

[8] P. Mancarella, "Cogeneration systems with electric heat pumps: Energyshifting properties and equivalent plant modelling," Energy Convers. Manag, vol. 50, pp. $1991-1999,2009$.

[9] P. Mancarella and G. Chicco, "Demand response from energy shifting in distributed multi-generation," IEEE Trans. Smart Grid, vol. 4, no. 4, Dec. 2013, pp. $1928-1938$.

[10] P. Mancarella and G. Chicco, "Integrated energy and ancillary services provision in multi-energy systems," in Proc. 2013 IREP SymposiumBulk Power System Dynamics and Control, 2013, pp. 1-19.

[11] M. B. Blarke and E. Dotzauer, "Intermittency-friendly and highefficiency cogeneration: operational optimisation of cogeneration with compression heat pump, flue gas heat recovery, and intermediate cold storage," Energy, vol. 36, no. 12, pp. 6867 -6878, 2011.

[12] M. Tasdighi, H. Ghasemi, A. Rahimi-Kian, "Residential microgrid scheduling based on smart meters data and temperature dependent thermal load modeling," IEEE Trans. Smart Grid, vol. 5, no. 1, pp. 349 $-357,2014$

[13] T. Capuder, P. Mancarella "Techno-economic and environmental modelling and optimization of flexible distributed multi-generation options," Energy, vol. 71, pp. 516 -533, 2014.

[14] A. K. Dixit and R. S. Pindy, Investment Under Uncertainty, Princeton University Press: 1994.

[15] E.A. Martínez Ceseña, J. Mutale and F. Rivas-Dávalos, "Real options theory applied to electricity generation projects: A review," Renew. Sustain. Energy Rev., vol. 19, pp. 573 -581, 2013.

[16] M. B. Blake, "Towards an intermittency-friendly energy system: Comparing electricity boilers and heat pumps in distributed cogeneration," Applied Energy, vol. 91, pp. 349-365, 2012.

[17] A. Fragaki, A. N. Anderson, D. Toke, "Exploration of economical sizing of gas engine and thermal store for combined heat and power plants in the UK," Energy, vol. 38, pp. 1659-1670, 2008.

[18] A. Piacentino, C. F. Cardona, R. Gallea and E Cardona, "A comprehensive tool for efficient design and operation of polygenerationbased energy $\mu$ grids serving a cluster of buildings. Part I: Description of the method," Applied Energy, vol. 11, pp.1024-1221, 2013.

[19] A. S. Siddiquie and K. Maribu, "Investment and upgrade in distributed generation under uncertainty," Energy Econ, vol. 31, no. 1, pp. 25 -37, 2009.

[20] G. Westner and R. Madlener, "Investment in new power generation under uncertainty: Benefits of CHP vs. condensing plants in a copula based analysis," Energy Econ, vol. 34, pp. 31 -44, 2012.

[21] M. Giuntoli and D. Poli, "Optimized thermal and electrical scheduling of a large scale virtual power plant in the presence of energy storages," IEEE Trans. Smart Grid, vol.4, no.2, pp.942 -955, June 2013.

[22] H. Karami, M. J. Sanjari, S. H. Hosseinian, G. B. Gharehpetian, "An optimal dispatch algorithm for managing residential distributed energy resources," IEEE Trans. Smart Grid, vol. 5, no. 5, pp. 2360 - 2367, 2014.

[23] G. K. J. Larsen, N. D. van Foreest, J. M. A. Scherpen, "Distributed MPC applied to a network of households with micro-CHP and heat storage," IEEE Trans. Smart Grid, vol. 5, no. 4, pp. 2106-2114, 2014.

[24] F. Rienze, P. Ahcin and G. Andersson, "Valuing investments in multienergy conversion, storage, and demand-side-management systems under uncertainty," IEEE Trans. on Sust. Energy, vol. 2, no.2, pp. $194-$ 202, 2011.

[25] E. Carpaneto, G. Chicco, P. Mancarella and A. Russo, "Cogeneration planning under uncertainty - Part I: Multiple time frame approach," Applied Energy, vol. 88, no. 4, pp. 1059-1067, 2011.

[26] E. Carpaneto, G. Chicco, P. Mancarella and A. Russo, "Cogeneration planning under uncertainty - Part II: Decision theory-based assessment of planning alternatives," Applied Energy, vol. 88, no. 4, pp. 1075 1083, 2011.

[27] A. H. van der Weijde and B. F. Hobbs, "The economics of planning electricity transmission to accommodate renewables: Using two-stage optimisation to evaluate flexibility and the cost of disregarding uncertainty," Energy Economics, vil. 34, no. 6, pp. 2089 -2101, 2012.

[28] L. Qiao Li, C. Tao, Y. Weng, R. Negi, F. Franchetti and M.D. Ilic, "An Information-Theoretic Approach to PMU Placement in Electric Power Systems," IEEE Trans. Smart Grid, vol.4, no.1, pp.446 -456, 2013.

[29] H. Long," Multistage transmission network planning with improved plant growth simulation algorithm," WIT Transactions on Information and Communication Technologies, vol 44. Pp. 649 -656, 2014.

[30] M. Rahmani, "Strategies to reduce the number of variables and the combinatioral search space of the multistage transmission expansion planning problem," IEEE Trans. on Power Syst., vol. 28, no. 3, pp. 2164 $-2173,2013$

[31] H. M. Nembhard and A. Mehmet, Real Options in Engineering design, Operations and Management. London: CRC Press, 2010.

[32] M.-A. Cardin and R. de Neufville, "A survey of state of the art methodologies and a framework for identifying and valuing flexible design opportunities in engineering systems," 2008. [Online]. Available: http://ardent.mit.edu/real_options/Real_opts_papers/WPCardindeNeufville2008.pdf 